\title{
Capillary-gravity wave resistance in ordinary and magnetic fluids
}

\author{
J. Browaeys, J.-C. Bacri, R. Perzynski \\ Groupe Ferrofluide associé à l'Université Denis Diderot \\ Laboratoire des milieux désordonnés et hétérogènes, UMR CNRS 7603 \\ Université Pierre et Marie Curie, case 78, 4 place Jussieu 75231 Paris Cedex 05, France \\ M. I. Shliomis \\ Department of Mechanical Engineering, Ben-Gurion University of the Negev, P.O.B. 653, \\ Beer-Sheva 84105, Israel
}

(January 7, 2018)

\begin{abstract}
Wave resistance is the drag force associated to the emission of waves by a moving disturbance at a fluid free surface. In the case of capillary-gravity waves it undergoes a transition from zero to a finite value as the speed of the disturbance is increased. For the first time an experiment is designed in order to obtain the wave resistance as a function of speed. The effect of viscosity is explored, and a magnetic fluid is used to extend the available range of critical speeds. The threshold values are in good agreement with the proposed theory. Contrary to the theoretical model, however, the measured wave resistance reveals a non monotonic speed dependence after the threshold.
\end{abstract}

PACS numbers: 47.35.+i, 68.10.-m, 75.50.Mm 
When an object is moved at the free surface of a fluid, it experiences a drag force which physically originates from: (a) bulk dissipation in a viscous boundary layer for low Reynolds numbers, and in the turbulent wake for high Reynolds numbers; (b) the emission of capillary-gravity surface waves. Such waves remove momentum from the perturbating object to infinity. The associated force that the object experiences is called wave resistance. For the convenient moderate speeds on which we focus in this paper it may overcome the bulk dissipation type drag.

Wave resistance has been studied for more than a century in the case of pure gravity waves [1], mainly because this topic has obvious naval applications [2]. In this case the variation of the wave resistance $R$ with the speed $V$ follows two régimes. A critical velocity $V_{c}^{\text {grav }}$ is imposed by the characteristic size $L$ of the ship: $V_{c}^{\text {grav }}=\sqrt{g L / 2 \pi}, g$ being the gravity acceleration. For $V<V_{c}^{\text {grav }}$ the wave resistance is very close to zero and behaves as $R \propto \sqrt{V-V_{c}^{g r a v}}$ for $V>V_{c}^{\text {grav }}$. This has recently been analyzed in terms of an imperfect bifurcation by one of the co-authors [3].

The case of capillary-gravity waves has been theoretically treated in a recent work of Raphaël and de Gennes [4]. Such waves are generated when the size of the perturbating object is small compared to the capillary wavelength $\lambda_{c}=2 \pi(\sigma / \rho g)^{1 / 2}$, where $\sigma$ is the surface tension of the free air-fluid interface and $\rho$ the density of the fluid. The dispersive properties of capillary-gravity waves are such as there exists a minimum phase speed $V_{c}=(4 \sigma g / \rho)^{1 / 4}$ at which waves are able to propagate. Since the pattern is stationary in the reference frame of the moving object, no wave can be emitted for $V<V_{c}$ [5], and therefore there is no wave resistance in that case. As it has been shown in [4], the wave resistance experiences a finite jump $R_{c}$ at $V=V_{c}$ and increases above $V_{c}$. The system is thus supposed to undergo a discontinuous bifurcation.

In order to check these theoretical predictions it is necessary to vary $V_{c}$ by means of $\rho$ and $\sigma$ variations. By adding a surfactant to water, $\sigma$ may be easily chosen between say 20 and $73 \mathrm{mN} / \mathrm{m}$. Consequently $V_{c}$ will merely vary from $17 \mathrm{~cm} / \mathrm{s}$ to $23 \mathrm{~cm} / \mathrm{s}$. A more efficient control parameter is thus needed. We here show that the action of a magnetic field on a magnetic fluid provides a means to tune the critical velocity from its maximum value $V_{c}^{H=0}=(4 \sigma g / \rho)^{1 / 4}$ down to 0 . Using a magnetic fluid, along with other regular fluids of different viscosities, we perform $R(V)$ measurements, as the problem has not been experimentally treated yet.

In a regular fluid, the wave emission process is controlled by the dispersion equation of capillary-gravity surface waves, $\omega^{2}=g k+\sigma k^{3} / \rho$, where $\omega$ is the circular frequency and $k$ the modulus of the wave vector. The condition for stationarity of the wave pattern in the frame of reference of the moving object is $\omega=k V \cos \theta$, where $\theta$ is the angle between the speed and wave vectors. Thus we obtain the following equation $\left(k_{c}=\sqrt{\rho g / \sigma}\right.$ is the capillary wavevector):

$$
\left(\frac{k}{k_{c}}\right)^{2}-2\left(\frac{V}{V_{c}} \cos \theta\right)^{2}\left(\frac{k}{k_{c}}\right)+1=0,
$$

which has no solution for $V<V_{c}$. For a moving Dirac Delta pressure distribution $P(x, y, t)=$ $p \delta(x-V t, y)$ the wave resistance is 国:

$$
R=\frac{p^{2}}{\pi \sigma} \int_{0}^{\arccos \frac{V_{c}}{V}} \cos \theta \frac{k_{+}(\theta)^{2}+k_{-}(\theta)^{2}}{k_{+}(\theta)-k_{-}(\theta)} d \theta
$$


where $k_{+}(\theta)$ and $k_{-}(\theta)$ are the two roots of Eq. (11). This formula remains valid as long as the characteristic size of the pressure distribution in experiments is much smaller than the capillary wavelength. In those conditions, close and above the threshold, the wave resistance has a finite value $R_{c}=p^{2} k_{c} / 2 \sqrt{2} \sigma$ and increases monotonically with speed (see inset of Fig. 1 and the uppermost curve in Fig. (1).

In order to measure $R(V)$ for the various fluids, they are placed into a circular channel dug into a Teflon covered aluminum dish. The latter is fixed to a shaft and rotated at constant rate, thus simulating a steady flow for the fluid. The radius of the channel is $20 \mathrm{~cm}$, its width is $2 \mathrm{~cm}$. A $4 \mathrm{~cm}$ wide channel is also used, showing no significant difference in the experimental results. The depth of the fluid is usually more than $1 \mathrm{~cm}$, ensuring the validity of the infinite depth approximation.

The disturbing object consists of a vertical bronze wire (radius $r=0.2 \mathrm{~mm}$ ) whose tip just touches the surface of the fluid (the wire is wetted by a few tenths of millimeters of fluid). The deflection of the wire is proportional to the horizontal force exerted on its free end (which is typically in the order of a micronewton). It is measured with an infrared optical sensor. The calibration of the sensor is obtained by tilting the base to which the wire is attached. A more detailed description of the measuring method will be published later.

Though no theory includes 3D viscous effects so far, we measure the wave resistance for different viscosities. To this purpose several mixtures of water and glycerol are used: the surface tension $\sigma$ and the densities $\rho$ of the mixtures are very close to one another (see Table 1) so that the impact of viscosity alone may be monitored in our experiments. The viscosities are measured with a standard Poiseuille viscometer.

Fig.11 displays the variation of the experimental drag $R_{\text {exp }}$ as a function of speed for the various mixtures. All the measurements are obtained by increasing and then decreasing the speed: there is no hysteresis. We may note that:

(a) There is a critical velocity at which the measured drag is discontinuous. That point validates an important feature of the Raphaël and de Gennes' theory 四. Besides, it has been checked that the drag discontinuity occurs at the very speed at which the wave pattern develops. The measured critical velocity $V_{c}$ is $23 \pm 0.5 \mathrm{~cm} / \mathrm{s}$ for pure water. It corresponds to a surface tension interval of $[65.1 ; 77.7] \mathrm{mN} / \mathrm{m}$ into which lies the tabulated value of pure water surface tension $72.75 \mathrm{mN} / \mathrm{m}$ at $20{ }^{\circ} \mathrm{C}$. For water/glycerol mixtures we obtained $V_{c} \approx 22.5 \mathrm{~cm} / \mathrm{s}$, that is compatible within experimental error bars with the surface tension of the mixtures (around $70 \mathrm{mN} / \mathrm{m}$ ).

(b) The experimental drag is not null below the critical velocity, all the more since the viscosity is high. The viscous drag $R_{\text {drag }}$ that is exerted over the immersed wire must be added to the wave resistance $R$ to account for the measured drag $R_{\exp }$. At a speed of 10 $\mathrm{cm} / \mathrm{s}$, the Reynolds number based on the approximate length of the wetted part of the wire $h \approx 0.4 \mathrm{~mm}$ is already equal to 40 (for water). A crude estimate of the viscous drag can still be given by the Stokes formula [7]: $R_{d r a g} \approx 6 \pi \eta h V$. We experimentally check that the viscous drag is indeed proportional to $h$; it can be seen in Fig.1 (dotted lines) that it is also proportional to $V$, at least for moderate enough speeds. The linear $R_{\text {drag }}$ dependence on viscosity $\eta$ is harder to assess because it is impossible to impose exactly the same $h$ from an experiment to another, the wetting of the wire being imperfect; nevertheless it is linear within the uncertainties over $h$. Inset of Fig.1 presents the $R(V)$ variations after 
subtraction of the viscous drag $R_{\text {drag }}$ for each sample. It is this quantity that has to be compared with the theoretical expression (2) (full line in the inset). It is clear from the inset that a pretransitional effect takes place, as the measured drag sharply increases just below the threshold (the higher the viscosity, the stronger the effect). A recent model [8] for 2D viscous wave resistance predicts such a feature.

(c) The amplitude of the wave resistance discontinuity at $V_{c}$ compares well with the theory. Assuming a perfect wetting of the wire by the fluid, the total force acting on the fluid is $p=2 \pi r \sigma$ ( $r$ is the radius of the wire). Thus an estimate of the wave resistance at the threshold is given by $R_{c}=\pi^{2} r^{2} \sqrt{2 \rho g \sigma}$. A comparison between expected values and what is

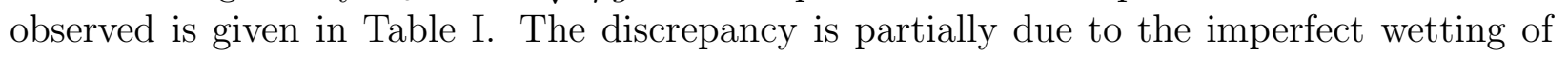
the fluid on the wire, which leads to overestimate the applied vertical force. On the other hand the drag values close to the threshold fluctuate a lot.

(d) The drag is a non-monotonic function of speed for $V>V_{c}$. In fact, it can be seen in inset of Fig. 1 that for $V>V_{c}$ the wave resistance $R$ first decreases as the speed increases, and then increases again for high enough speeds. This feature is not predicted by the current theory, which anyway overestimates the actual drag. Moreover it is unlikely a viscosity effect since it is as much marked as the viscosity is low. It is possibly a general feature of such a flow, and in this case the theory should be revised to include viscosity and non-linear aspects.

In a magnetic fluid the dispersion equation of capillary-gravity surface waves is modified with allowance for a vertical uniform magnetic field [11]:

$$
\omega^{2}=g k+\frac{\sigma k^{3}}{\rho}-\mu_{0} \frac{\left(\mu_{r}-1\right)^{2}}{\mu_{r}\left(\mu_{r}+1\right)} \frac{H^{2} k^{2}}{\rho},
$$

where $H$ is the magnetic field, $\mu_{r}$ the relative magnetic permeability of the magnetic fluid (assumed to be constant [11]) and $\mu_{0}$ the vacuum magnetic permeability. For a given wave vector, an increase of the field intensity lowers the frequency of the waves. The frequency drops to zero when $H$ reaches a certain critical value $H_{*}$ defined by:

$$
H_{*}^{2}=2 \frac{\mu(\mu+1)}{(\mu-1)^{2}} \frac{\sqrt{\rho g \sigma}}{\mu_{0}}
$$

For $H>H_{*}$ the surface becomes unstable: the Rosensweig instability, sometimes called the peak instability, develops yielding an hexagonal array of peaks [6]. The condition for stationarity implies that $k$ must be a solution of:

$$
\left(\frac{k}{k_{c}}\right)^{2}-2\left(\left(\frac{V}{V_{c}} \cos \theta\right)^{2}+\left(\frac{H}{H_{*}}\right)^{2}\right)\left(\frac{k}{k_{c}}\right)+1=0 .
$$

Real solutions exists if and only if:

$$
V>V_{c}^{H} \text { with } V_{c}^{H}=V_{c} \sqrt{1-\left(\frac{H}{H_{*}}\right)^{2}}
$$

therefore a steady vertical magnetic field should allow the tuning of the critical velocity at which waves (and wave resistance) appear. 
The wave resistance, following Eq.(2) and Eq.(5)), is given by the following integral:

$$
\begin{gathered}
R^{H}(V)=\frac{p^{2} k_{c}}{\pi \sigma} \int_{0}^{\arccos \frac{V_{c}^{H}}{V}} \cos \theta\left(2 B(V, \theta)^{\frac{1}{2}}+B(V, \theta)^{-\frac{1}{2}}\right) d \theta \\
\text { where } B(V, \theta)=\left(\left(\frac{V}{V_{c}} \cos \theta\right)^{2}+\left(\frac{H}{H_{*}}\right)^{2}\right)^{2}-1
\end{gathered}
$$

Just above the threshold, the wave resistance has the finite value :

$$
R_{C}^{H}=\frac{p^{2} k_{c}}{2 \sqrt{2} \sigma}\left(1-\left(\frac{H}{H_{*}}\right)^{2}\right)^{-\frac{1}{2}} .
$$

Another experiment is conducted using a water based magnetic fluid synthesized according to the Massart method [9]. Its critical field $H_{*}$ is $9.15 \mathrm{kA} / \mathrm{m}$ and its surface tension of 60 $\mathrm{mN} / \mathrm{m}$ doesn't depend on the magnetic field. Other caracteristics are given in table $\mathbb{1}$. The experimental critical values $V_{c}^{H}$ and $R_{c}^{H}=R_{e x p}\left(V_{c}^{H}\right)-R_{d r a g}\left(V_{c}^{H}\right)$ are both plotted versus the normalized magnetic field $H / H_{*}$ in Fig. 2 and Fig. 3, and are compared to theoretical predictions (6) and (9).

The theoretical expression (6) of $V_{c}^{H}$ (Fig. 2) remarkably fits the data points - note that there is no adjustable parameters. A data point lies outside the curve, but this is probably related to an imperfect magnetic wetting phenomenon. As the magnetic field gets closer to the peak instability threshold value $H_{*}$, the fluid "climbs" onto the wire, producing a much higher viscous drag, a situation which gets away from our inviscid linear theoretical analysis. This also explains the discrepancy in Fig. 3 between experimental and theoretical $R_{C}^{H}$ values. We do not account for the force that the magnetic field is exerting at the meniscus close to the wire. Indeed, the very shape of the meniscus creates a non homogeneous magnetic field which results in a force that sucks the magnetic fluid up and changes the shape of the meniscus. Only advanced numerical simulations would allow to compute the net force added [10].

Fig. 1 presents the results obtained for different magnetic fields in a reduced representation $R / R_{c}^{H}=f\left(V / V_{c}^{H}\right)$ with $R^{H}=R_{e x p}-R_{d r a g}$. It also gives a comparison to the theoretical predictions of Eq.(17). As it was pointed out with regular viscous fluids, the theoretical variations of $R / R_{c}^{H}$ lie above the data points, except for $H \approx H_{*}$. Then the experimental data and the theory are very comparable. The present theoretical description thus gives a correct general trend for the influence of the field on the wave resistance.

In conclusion, for the first time a capillary-gravity wave resistance measurement is performed on fluids of various viscosities. A drag discontinuity is always observed for a critical velocity $V_{c}$. Thanks to a magnetic fluid the critical velocity range is experimentally extended. In all cases the measured critical velocities and the critical values of the resistance are in good accordance with the developped models. If an inviscid theory is correct at the threshold, there are some discrepancies for $V>V_{c}$ such as a non-monotonic behavior of the wave resistance. Viscosity and non linear aspects should be taken into account in further works. Finally, in order to get rid of the viscous drag that is always present in our experiments, another mode of disturbance is to be envisaged, such as a small magnet placed just above the free surface of a flowing magnetic fluid. 
We wish to thank J. Servais and P. Lepert for their technical assistance, S. Neveu for providing us with the ferrofluid sample and E. Raphaël for helpful comments. 


\section{REFERENCES}

[1] Lord Kelvin, Proc. R. Soc. London A 42, 80 (1887).

[2] A.A Kostyukov, Theory of Ship Waves and Wave Resistance (Effective Commun. Inc., Iowa City, 1968).

[3] M. I. Shliomis and V. Steinberg, Phys. Rev. Lett. 79, 4178 (1997).

[4] E. Raphaël and P.-G. de Gennes, Phys. Rev. E, 533448 (1996).

[5] J. Lighthill, Waves ind Fluids, (Cambridge University Press, Cambridge, 1996).

[6] M. D. Cowley and R. E. Rosensweig, J. Fluid Mech. 30, 671 (1967).

[7] L. D. Landau and E. M. Lifshitz, Fluid Mechanics, 2nd ed. (Pergamon Press, New York, 1987).

[8] D. Richard and E. Raphaël, to be published in Europhys. Lett..

[9] R. Massart, IEEE Trans. Magn. 17, 1247 (1981).

[10] A. G. Boudouvis and L. E. Scriven, J. Magn. Magn. Mater. 122, 254 (1993).

[11] J. Browaeys, J.-C. Bacri, C. Flament, S. Neveu and R. Perzynski, Eur. Phys. J. B 9, 335 (1999). 


\section{FIGURES}

FIG. 1. Experimental drag $R_{\exp }$ as a function of speed $V$ for different water glycerol mixtures. For readability purpose only a few error bars are plotted. Dotted lines : linear viscous drag $R_{d r a g}$ for each viscosity. Inset : wave resistance $R=R_{e x p}-R_{d r a g}$ as a function of $V$. Same symbols as in the main figure. Full line : theoretical expression from Eq. (2).

FIG. 2. Reduced critical speed $V_{c}^{H} / V_{c}^{H=0}$ at which wave resistance appears in function of the applied reduced magnetic field $H / H_{*}$. The straight line represents the theoretical law given by Eq.(6). There is no adjustable parameter.

FIG. 3. Drag at threshold $R_{c}^{H}$ as a function of the reduced magnetic field $H / H_{*}$. The full line represents the theoretical law as given by Eq.(9). There is no adjustable parameter.

FIG. 4. Measured wave resistance $R^{H}=R_{e x p}^{H}-R_{d r a g}^{H}$ as a function of reduced speed $V / V_{c}^{H}$ for different reduced magnetic fields $H / H_{*}$. The theoretical curves are derived from Eq. (77,8). The uppermost curve describes the wave resistance of a regular non-magnetic fluid. 


\section{TABLES}

TABLE I. Experimental drag discontinuity at the threshold compared to the theoretical predictions of [4], for various water glycerol mixtures and an aqueous magnetic fluid (MF).

\begin{tabular}{lccccc}
\hline \hline Glycerol mass fraction $(\%)$ & 60 & 44.5 & 30 & 0 & MF \\
Viscosity $(\mathrm{mPa} . \mathrm{s})$ & 12.5 & 5.1 & 2.6 & 1.0 & 7.0 \\
Density $\left(\mathrm{g} / \mathrm{cm}^{3}\right)$ & 1.16 & 1.13 & 1.09 & 1.00 & 1.56 \\
\hline Theory $(\mu \mathrm{N})$ & 3.9 & 3.8 & 3.8 & 3.7 & 4.2 \\
Experience $(\mu \mathrm{N})$ & 2.9 & 2.6 & 4.0 & 3.6 & 4.0 \\
Uncertainty $(\mu \mathrm{N})$ & 0.3 & 0.3 & 0.4 & 1.8 & 1.0 \\
\hline \hline
\end{tabular}




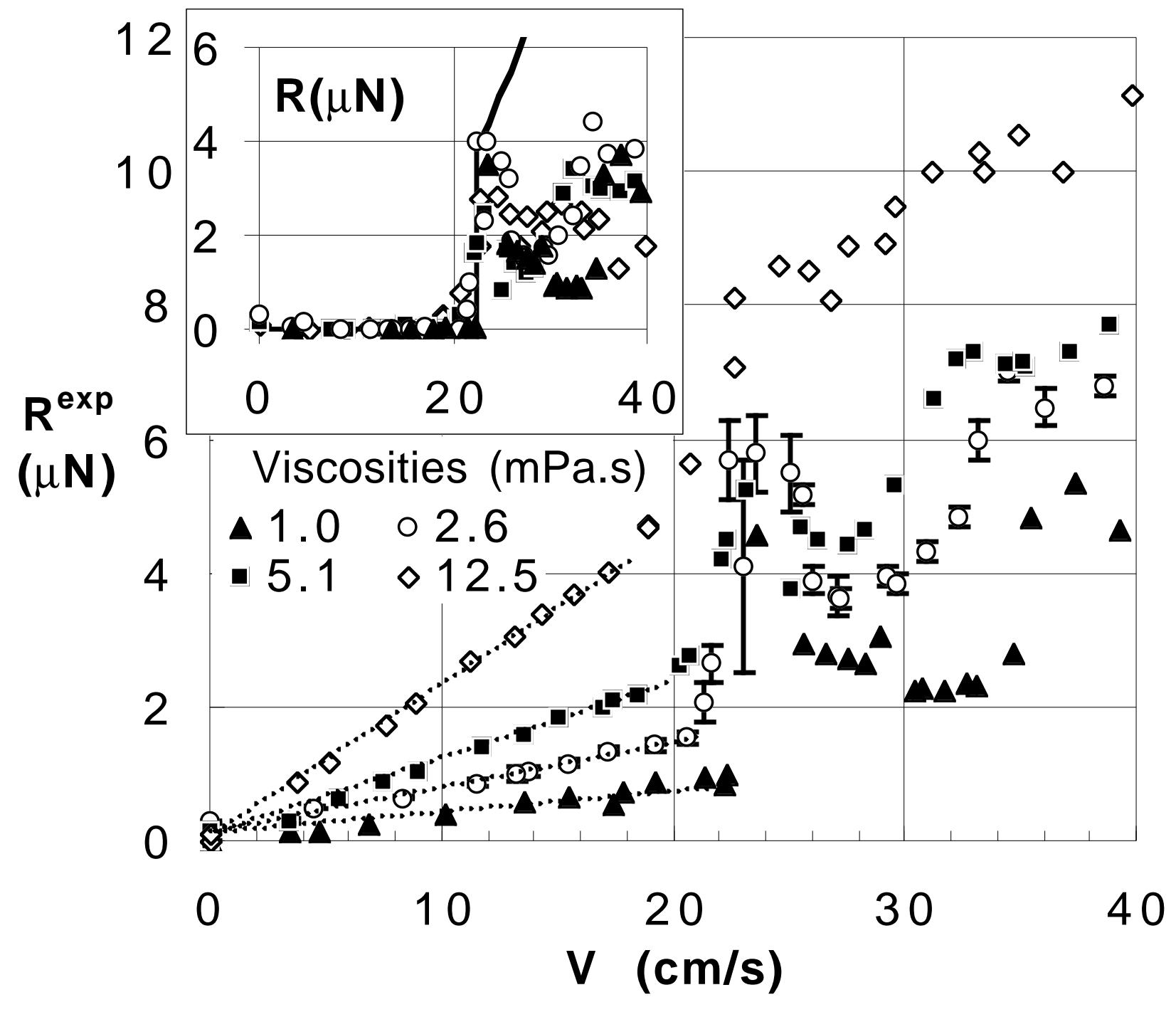




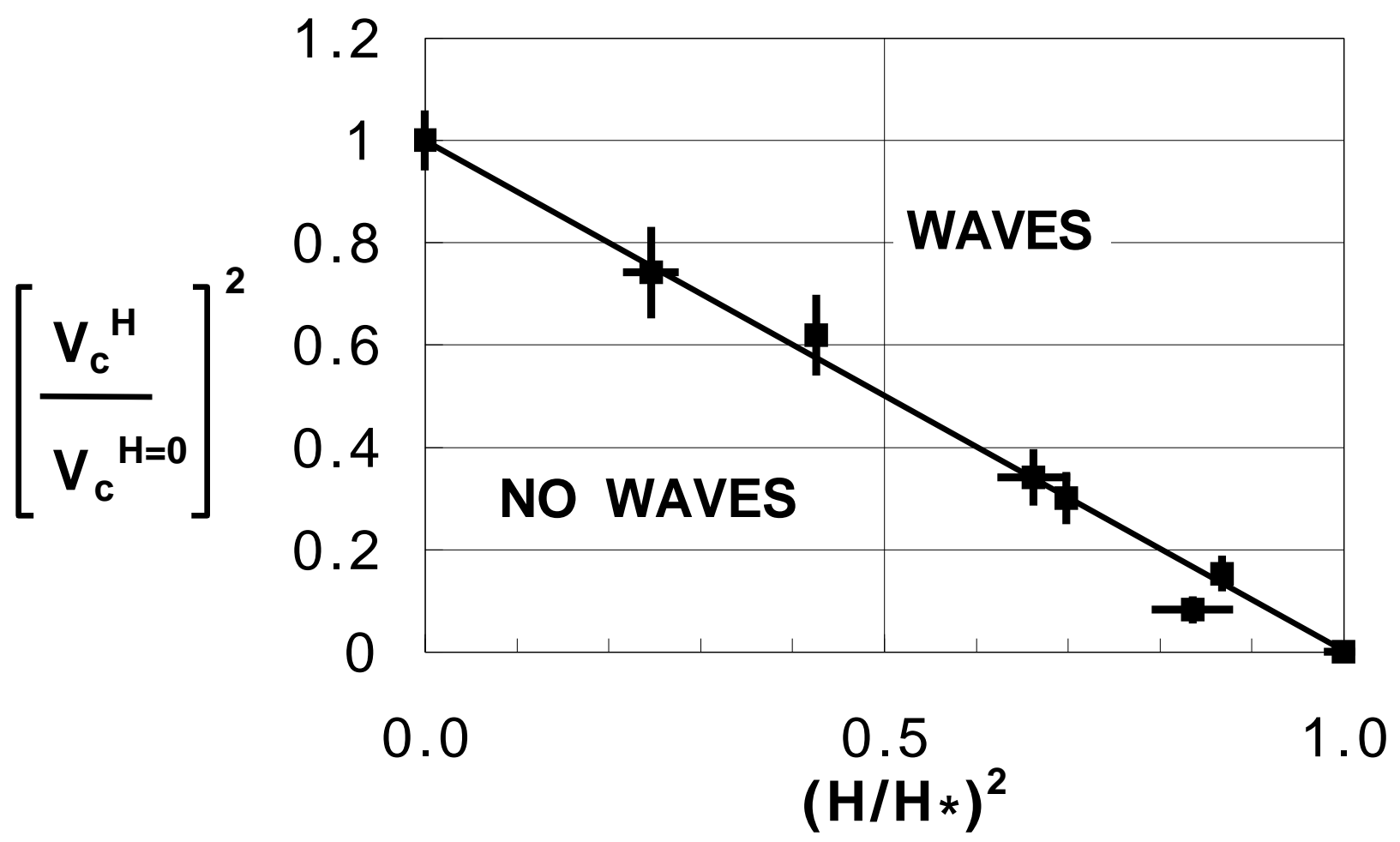




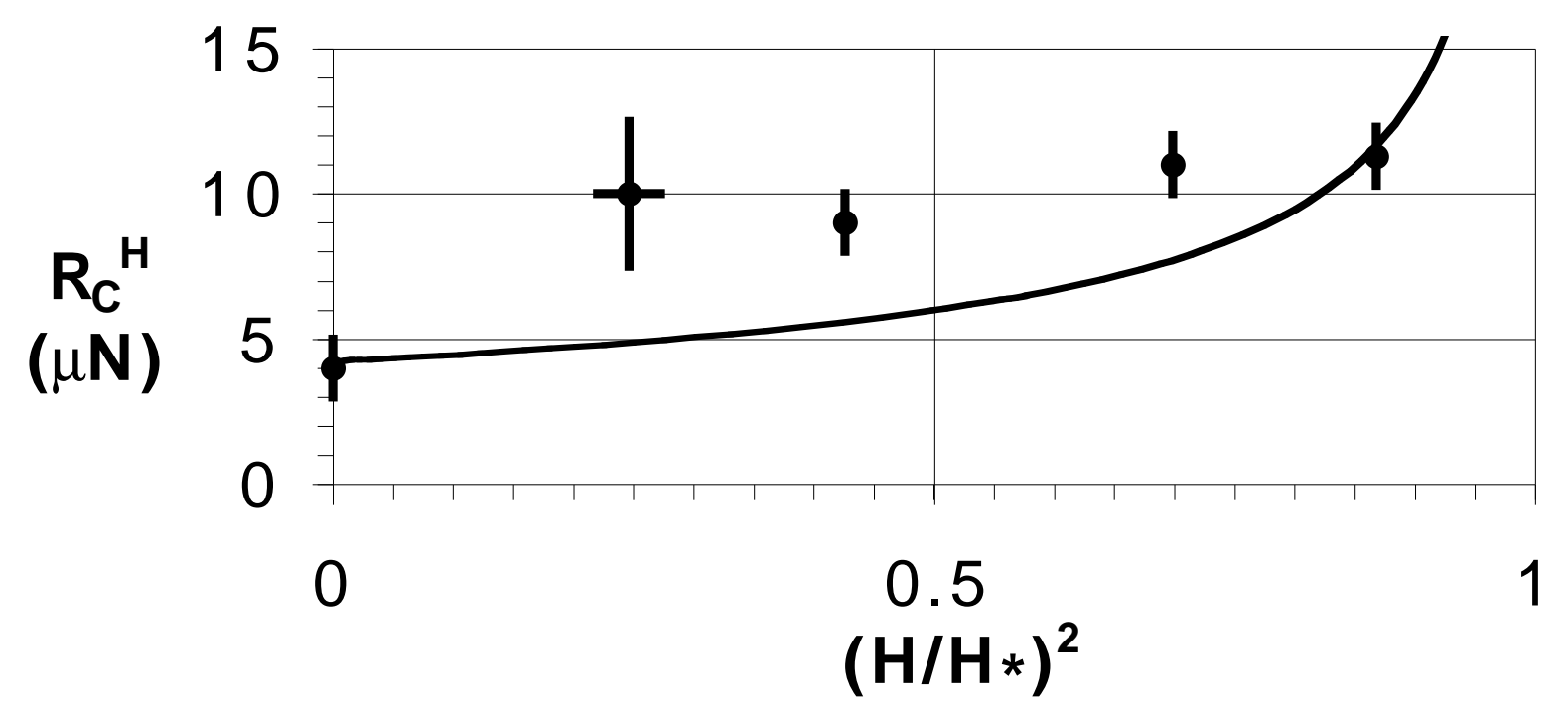




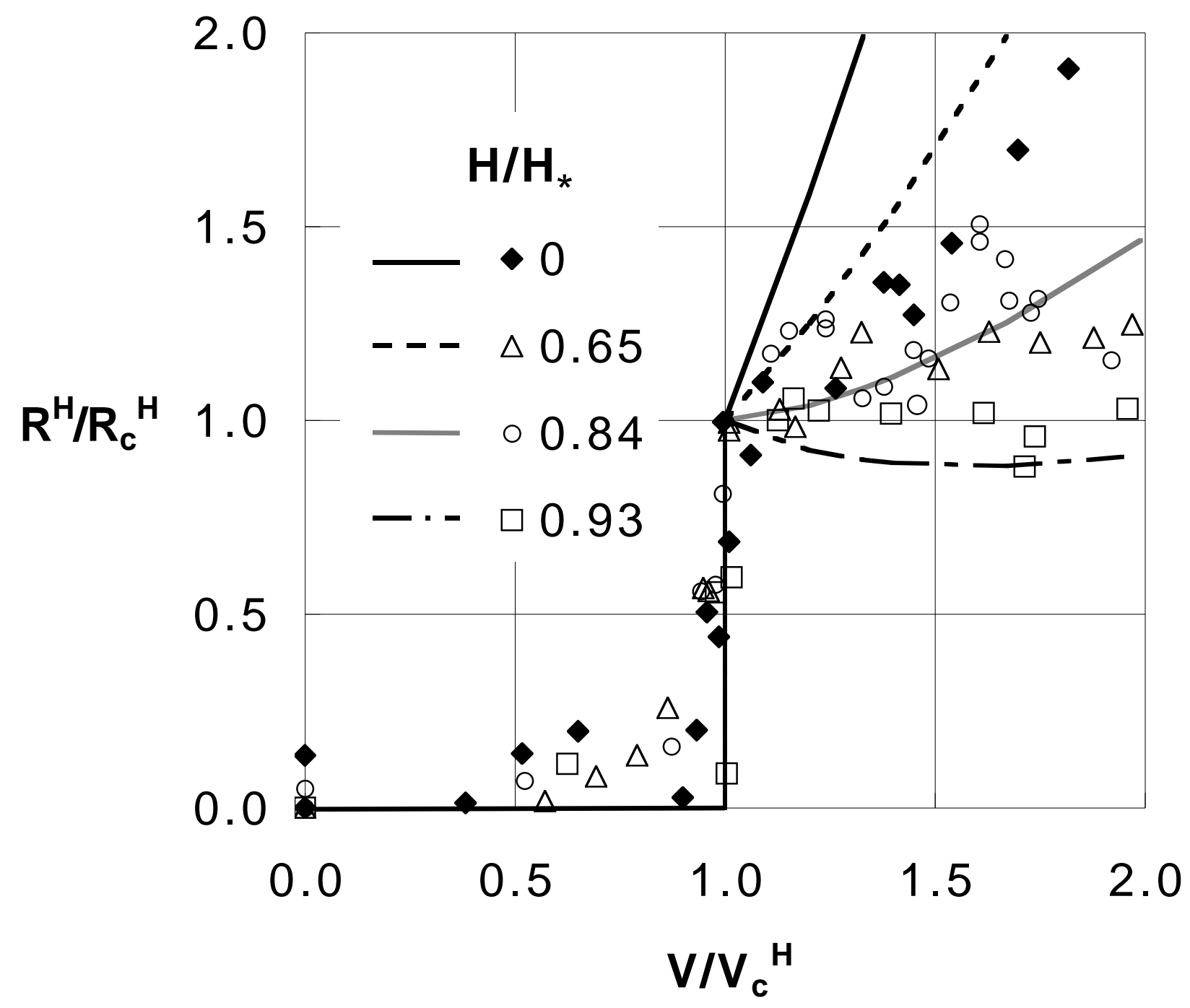

